\title{
Studies on Fluorescence Efficiency and Photodegradation of Rhodamine 6G Doped PMMA Using a Dual Beam Thermal Lens Technique
}

\author{
ACHAMMA KURIAN*, NIBU A GEORGE, BINOY PAUL, \\ V. P. N. NAMPOORI and C. P. G. VALLABHAN \\ International School of Photonics, Cochin University of Science \& Technology, \\ Cochin 682022, India
}

(Received 11 September 2002)

\begin{abstract}
In this paper we report the use of the dual beam thermal lens technique as a quantitative method to determine absolute fluorescence quantum efficiency and concentration quenching of fluorescence emission from rhodamine 6G doped Poly(methyl methacrylate) (PMMA), prepared with different concentrations of the dye. A comparison of the present data with that reported in the literature indicates that the observed variation of fluorescence quantum yield with respect to the dye concentration follows a similar profile as in the earlier reported observations on rhodamine $6 \mathrm{G}$ in solution. The photodegradation of the dye molecules under $\mathrm{cw}$ laser excitation is also studied using the present method.
\end{abstract}

Key words: Thermal lens; Fluorescence quantum yield; Photostability; Dye doped polymer

\section{INTRODUCTION}

From the mid 60 s, dye lasers have been attractive sources of coherent tunable radiation because of their unique operational flexibility. The salient features of dye lasers are their tunability, with emission from near ultraviolet to the near infrared. High gain, broad spectral bandwidth enabled pulsed and continuous wave operation. Although dyes have been demonstrated to lase in the solid, liquid or gas phases, liquid solutions of dyes in suitable organic

\footnotetext{
* Corresponding author. Catholicate College, Pathanamthitta 689645, India. E-mail: achammakurian@rediffmail.com
} 
solvents have been the most frequently used laser media. This is because the active medium can be obtained in high optical quality, the cooling is achieved by using a flow system and the medium is self-repairable to a certain extent. Nevertheless, the use of liquid solutions in dye lasers entails a number of inconveniences, mainly related to the need of employing large volumes of organic solutions of dyes that are both toxic and expensive. Furthermore, each dye has a limited range of tunability $(5 \mathrm{~nm}$ to $20 \mathrm{~nm})$ so that the dye has to be changed for different wavelength regions. This, together with the need for complex and bulky cell designs for the continuous circulation of the solution, has restricted the use of these laser systems outside the laboratory.

From the early days of development of dye lasers, attempts were made to overcome the problems posed by dye solutions, by incorporating dye molecules into solid matrices. A solid-state dye laser avoids the problems of toxicity and flammability and they are compact, versatile and easy to operate and maintain. The first observations of stimulated emission from solid matrices doped with organic dyes were reported as early as 1967 and 1968 by Soffer and Mcfarland [1] and Peterson and Snavely [2], respectively. Since laser dyes were in most cases merely dispersed, the results were not very encouraging due to the low lasing efficiencies and fast dye photodegradation. In recent years, the synthesis of high performance dyes and the implementation of new ways of incorporating the organic molecules into the solid matrix have resulted in significant advances towards the development of practical tunable solid-state lasers [3-12]. The use of a synthetic polymer host presents advantages as these materials show much better compatibility with organic laser dyes and are amenable to inexpensive fabrication techniques [13]. These polymers provide an opportunity for the production of active elements that can effectively control the characteristics of laser radiation. Hence, adequate knowledge of thermal and optical properties of dye doped polymers is important in identifying suitable laser media.

Fluorescence quantum efficiency is one of the most important optical properties of fluorescent materials and it is the essential parameter in determining the lasing characteristics of the active laser medium. Most methods presently followed use conventional techniques for determining the absolute quantum yield of fluorescence by comparing emissions from fluorescent solutions with radiation scattered by a standard scatterer. In these kinds of measurements it is necessary to introduce corrections for the spectral sensitivity of the detection system. Pure optical measurements also demand calibration of the detector. The use of fluorescence lifetime is also inadequate, especially for multilevel energy systems, as it depends both on the value of the nonradiative lifetime and fluorescence decay time. Even after the various corrections for system geometry, re-absorption, polariza- 
tion etc., the accuracy of the quantum yield values obtained from photometric measurements is rather poor [14]. It is in this context that an alternate technique using photothermal effect is employed to evaluate the absolute quantum efficiency of luminescent samples.

The introduction of photothermal techniques to measure the quantum efficiency has brought new perspectives to obtain more accurate values for this parameter. The basis of the photothermal effect is a photo-induced change in the thermal state of the sample. Thermal lens spectroscopy is one of such techniques, which is fast, non-contacting, non-destructive and can be performed in a low frequency range. When a medium is illuminated with a laser beam, some of the energy is absorbed by the molecules in the ground state which are excited to higher energy states. After the absorption of the photon the excess energy attained by the molecule can be dissipated in many ways. The non-radiative decay process causes heating of the sample which creates a refractive index gradient in the medium, and this produces a lens like optical element called the thermal lens (TL). The propagation of the probe beam through the thermal lens will result in either a spreading or a focusing of the beam center, depending upon the temperature coefficient of the thermal expansion. The TL effect has been exploited for a number of measurements such as determination of absorptivities as low as $10^{-7} \mathrm{~cm}^{-1}$, evaluation of triplet quantum yield in solid and liquid phases, the study of multiphoton processes, thermal diffusivity of various solvents, calorimetric trace analysis, food stuff analysis and electronic energy transfer studies [15-21].

The application of the photothermal lensing effect for the measurement of fluorescence quantum yields was first introduced by Brannon and Madge [22]. Since then, the technique has been used to determine this parameter in fluorescent solutions [23] and fluorescent polymers [24]. In these experiments a single beam configuration has been used to evaluate this parameter. In the present experimental setup a dual beam thermal lens technique is used to find the fluorescence quantum efficiency of dye doped polymers since this method is more sensitive than the single beam configuration [25].

In the present investigation, the absolute value of fluorescence quantum yield, the effect of concentration of the dye on its fluorescence efficiency in the matrix and the mechanisms responsible for the photostability of the dye in the matrix are studied.

\section{MATERIALS AND METHOD}

Methyl methacrylate (MMA) (Merck) was washed three times with (2 vol\%) aqueous sodium hydroxide to remove the inhibitor and then twice 
with distilled water. An accurately weighed amount of rhodamine 6G (Exciton) was dissolved in a mixture of MMA and ethanol taken in the ratio $4: 1$ to give a concentration of $1.5 \times 10^{-3} \mathrm{moll}^{-1}$. Since rhodamine $6 \mathrm{G}$ has limited solubility in MMA ethyl alcohol was added. Apart from being a good solvent ethyl alcohol enhances the value of dielectric constant, which increases the conversion efficiency. From the stock solution, sample solutions with different concentrations ranging from $1.5 \times 10^{-3} \mathrm{moll}^{-1}$ to $1 \times 10^{-4} \mathrm{moll}^{-1}$ were prepared. Then $1.5 \mathrm{gm} / 1$ of benzoyl peroxide $(\mathrm{CDH}$ chemicals) $(0.15 \%$ by weight $)$, which is a free-radical initiator, was added to each sample solution. The mixture was then taken in glass bottles and kept in a constant temperature bath maintained at $500^{\circ} \mathrm{C}$ for polymerization. After about 48 hours completely polymerized samples were taken out and kept for one week for drying. Care was taken to see that the dye is homogeneously distributed in the polymer matrix. The dried samples were then cut into pieces of $2 \mathrm{~mm}$ thickness. The disc shaped samples thus obtained were initially lapped using different grades of silicon carbide to obtain a smooth surface quality. This was followed by a polishing process employing fine grade Alumina $\left(\mathrm{Al}_{2} \mathrm{O}_{3}\right)$ powder. The various grades for polishing include 1, 0.5 and 0.3 microns, on TEXMET polishing cloth. All these ensured minimum loss due to scattering as all the samples were made to good optical finish. Plain undoped PMMA samples were also prepared under the same experimental conditions and these were used as reference samples to record the optical absorption spectrum.

The experimental setup of the dual beam thermal lens technique employed in the present investigation is shown in Figure 1. Laser radiation at $532 \mathrm{~nm}$ wavelength from a Diode Pumped Nd: $\mathrm{YVO}_{4}$ (Uniphase BWT50) laser was used as the pump beam to generate the thermal lens in the medium. Radiation of wavelength $632.8 \mathrm{~nm}$ from a low power $(1 \mathrm{mw})$ intensity stabilized He-Ne laser source was used as the probe beam. The pump beam was intensity modulated at $5 \mathrm{~Hz}$ using a mechanical chopper (EG\&G 192). Sample, in the form of a disc having a thickness of $2 \mathrm{~mm}$, was kept in the pump beam path. The probe beam was made to pass collinearly through the sample using a dichroic mirror. An optical fibre mounted on XYZ translator served as the finite aperture. The other end of the fibre was coupled to a monochromator-PMT assembly that was set at $632.8 \mathrm{~nm}$. The signal output from PMT was processed using a dual phase lock-in amplifier (Stanford Research systems SR-850).

The absorption spectra of the sample having different concentrations were recorded using a UV-VIS-IR (JASCO-570/UV/VIS/NIR) spectrophotometer. For the fluorescence study, the front surface emission was collected and focused by a lens to the entrance slit of a $1 \mathrm{~m}$ Spex mono- 


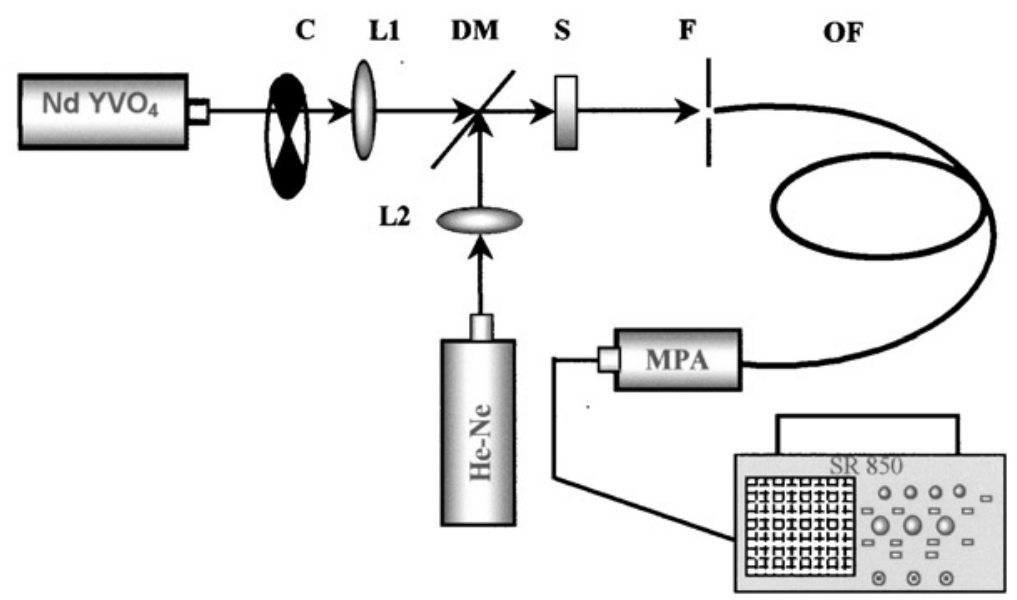

Lock-in amplifier

FIGURE 1 Schematic diagram of the experimental set up. (C-chopper, $\mathrm{L}_{1}, \mathrm{~L}_{2}-$-lens, DMdichoric mirror, S-sample, OF-optic fibre, MPA-monochromator-PMT assembly.

chromator, which was coupled to a PMT having an S20 cathode. The PMT output was fed to a lock-in amplifier. The emission wavelength was scanned in the specified region $(520-620 \mathrm{~nm})$.

\section{RESULTS AND DISCUSSION}

The absorption spectra of the solid samples at different concentrations are given in Figure 2. At low concentration dye-dye interaction is negligible because of the large average distance between them. As we increase the concentration, absorption spectra contain contributions from monomers and aggregates which make the spectrum broader. Figure 3 shows the fluorescence spectrum of the polymer sample for different dye concentrations. Fluorescence of a molecule depends on the structure and environment of the molecule such as interaction with solvent and other dissolved compounds in the matrix, the temperature, the $\mathrm{pH}$ and concentration of the fluorescing species. Dyes dissolve completely as monomers at low concentrations and obviously the dye-dye interaction and the intermolecular interactions between dye molecules and macromolecules are negligible. Hence, at very low concentration, fluorescence intensity increases linearly with increasing concentration. At higher concentrations fluorescence intensity reaches a limiting value and then decreases with further increase 


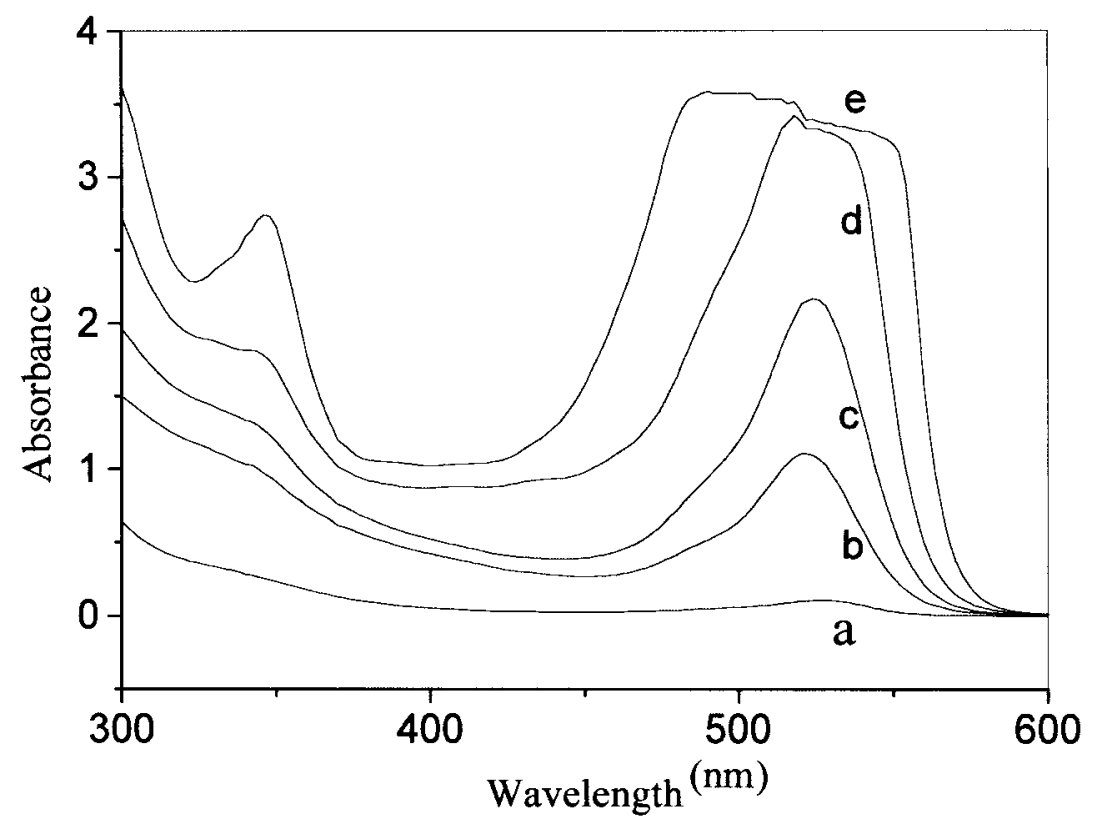

FIGURE 2 Absorption spectra of rhodamine 6G doped PMMA for various concentrations in $\mathrm{m} \mathrm{moll} 1^{-1}$, (a) 0.1 (b) 0.4 (c) 0.54 (d) 0.7 (e) 0.8 .

in concentration. This can be related to the phenomenon of re-absorption and re-emission, which ultimately reduces fluorescence emission. With increasing dye concentration the formation of dimers and higher aggregates decreases the fluorescence emission by a combination of monomerdimer/higher aggregates energy transfer and absorption of radiation by non-fluorescent dimers/higher aggregates [26]. This transfer of energy between molecules by collisional mechanisms makes the nonradiative part prominent and hence fluorescence decreases. However, in the present case, re-absorption and emission are the probable causes for the reduction in fluorescence intensity at higher concentrations. The observed peak shift in the fluorescence spectra is a consequence of re-absorption and re-emission. Since the low frequency tail of the absorption spectrum of the dye molecule overlaps with the high frequency end of its fluorescence spectrum, the fluorescence from the excited state dye molecule is reabsorbed by the ground state molecule which shifts the fluorescence peak to lower energies. Absorption saturation would lead to a similar red shift, depending on the medium. However, in general the red shift in dyes is not due to saturation. 


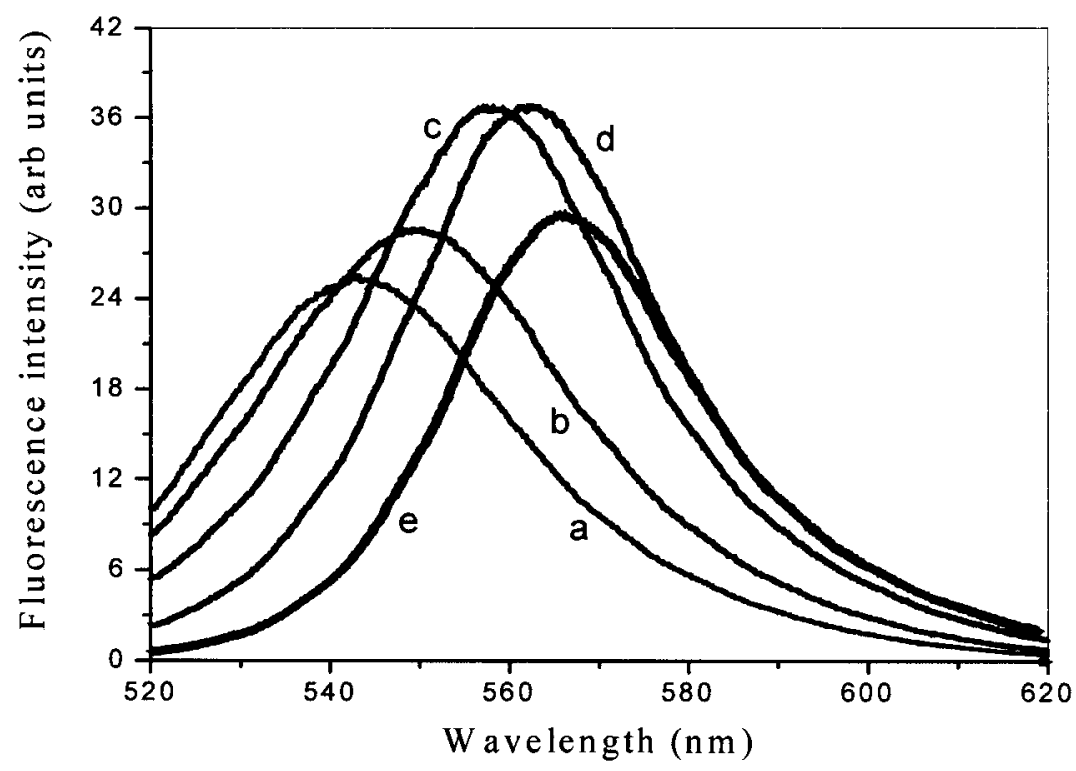

FIGURE 3 Fluorescence spectra of rhodamine 6G doped PMMA for various concentrations in $\mathrm{m} \mathrm{mol}^{-1}$ (a) 0.4 (b) 0.54 (c) 0.7 (d) 0.8 (e) 0.9 .

The absolute fluorescence yield of rhodamine $6 \mathrm{G}$ is determined using the equation [22]

$$
Q_{f}=\left(1-\frac{\eta}{\eta_{a}}\right) \frac{\lambda_{f}}{\lambda}
$$

Here the ratio of the fluorescence peak wavelength $\lambda_{f}$ to excitation wavelength $\lambda$ takes into account of the stokes shift. $\eta_{a}$ is directly proportional to TL signal corresponding to the concentration at which the fluorescence intensity is quenched completely. The thermal lens signal $\eta$ has been measured as the variation of light intensity at far field at the centre of the probe beam.

The variation of quantum yield with concentration is shown in Figure 4. The laser power is kept constant at $5 \mathrm{~mW}$ in this experiment. The quantum yield closely depends on the environment of the fluorescing molecule and on processes like internal conversion, intersystem crossing and solutesolute interaction. These parameters strongly depend on the excitation source, solvent characteristics and concentration of the dye. 


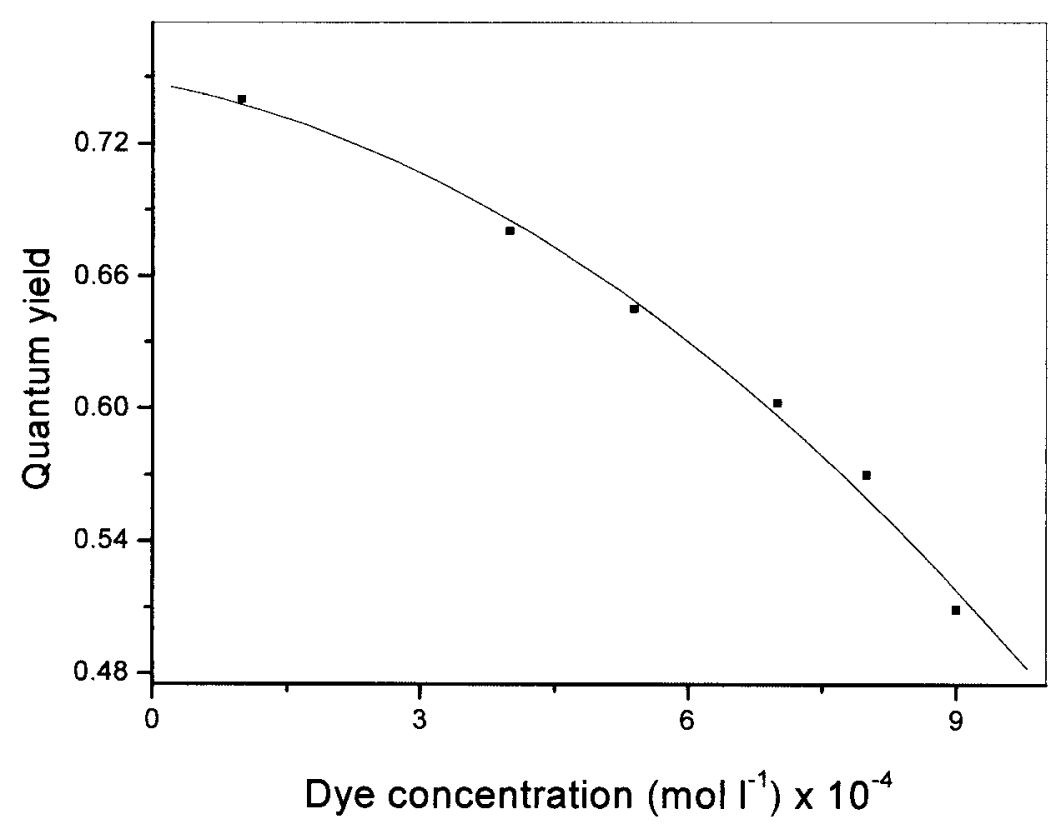

FIGURE 4 The variation of quantum yield of rhodamine 6G doped PMMA with concentration for a power of $5 \mathrm{~mW}$.

The plot clearly reveals a decrease in fluorescence quantum yield at higher concentrations. This is a direct indication of the fact that nonradiative processes become significant at higher concentrations, which contribute to enhanced thermal lensing. One of the factors which reduces the fluorescence quantum yield is the re-absorption of emitted radiation by ground state molecules. This also enhances nonradiative relaxation processes between various vibronic levels of the dye molecules. One of the major mechanisms that may quench the fluorescence emission in $\mathrm{cw}$ mode excitation is relaxation to the triplet state. Triplet state absorption is sensitive to the excitation wavelength. Korobov et al. studied the triplet states of rhodamine $6 \mathrm{G}$ by means of flash photolysis [27]. Their studies reported negligible triplet-triplet absorption for the wavelength $532 \mathrm{~nm}$. Thus the influence of triplet-triplet absorption on the excitation wavelength used in our studies could be safely neglected. Moreover, triplet-triplet absorption occurs at high laser intensities [28,29]. Since we carried out the experiment at low laser powers the contribution to thermal lens signal due to excited triplet states relaxation is ruled out. Generation of dimers, excimers and 
other aggregates will also contribute to the decrease in fluorescence quantum yield. Dimers and higher aggregates can quench monomer emission by collisional or long-range nonradiative energy transfer.

Apart from their use as active laser media, dye doped polymer composites have been considered as candidates for new electro-optic materials, logic gates, photo-detectors, holographic recording and solar concentrators $[30,31]$. In this regard it is highly desirable to study the photostability of doped samples. Figure 5 describes the time evolution of TL signal for different pump powers. Increase in TL signal at low powers ( 3 and $6 \mathrm{~mW})$ is due to residual thermal lens signal due to the previous cycles of the intensity modulation. The thermal lens decay due to the previous cycle is not completed due to characteristic signal response time, $t_{c}$.

The time dependent decrease in the TL signal is obviously due to the photo-induced chemical changes in the present sample. It can be seen that the dye molecules embedded in the PMMA matrix do not undergo anychange in chemical properties at very low laser powers, such as $3 \mathrm{~mW}$ and $6 \mathrm{~mW}$. However, the gradual decrease in the TL signal for laser powers

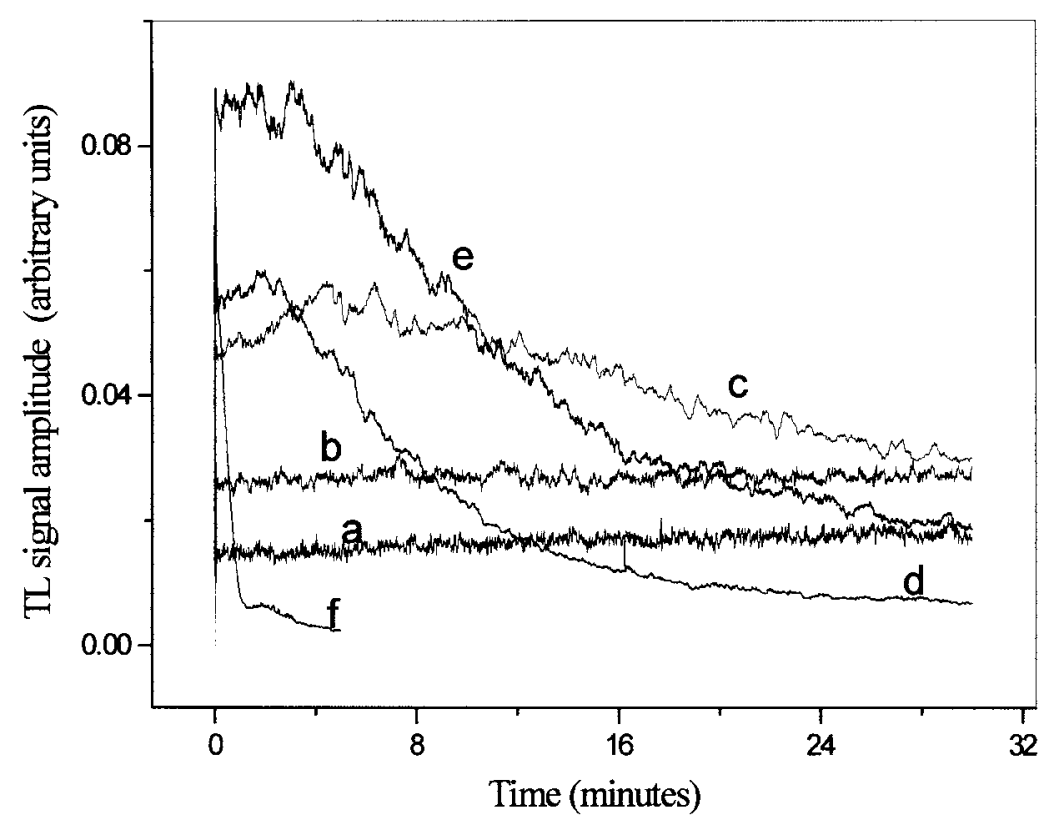

FIGURE 5 The variation of thermal signal strength with time for laser powers (a) $3 \mathrm{~mW}$ (b) $6 \mathrm{~mW}$ (c) $15 \mathrm{~mW}$ (d) $20 \mathrm{~mW}$ (e) $28 \mathrm{~mW}$ (f) $42 \mathrm{~mW}$. The concentration of the sample is 
$15 \mathrm{~mW}$ and above, reflects the photodegradation of rhodamine $6 \mathrm{G}$ molecules. From Figure 5 it is very clear that the rate of this reaction increases with increase in the pump power. The saturation in the TL signal is an indication of complete destruction of the dye molecules in the pump beam path. The photobleaching of the dye was found to result in a permanent colour change, which indicates that the reaction is an irreversible one. This is in accordance with the earlier reported photoacoustic measurements on dye doped PMMA [32]. The completely bleached portion of the sample is almost as transparent as an undoped PMMA sample (Fig. 6), which implies that the photochemical reaction product of rhodamine $6 \mathrm{G}$ does not absorb in the visible region. The photochemical degradation of rhodamine $6 \mathrm{G}$ occurs only in the presence of suitable optical radiation, which produces large local increases in temperature and thermal destruction of the dye molecules. Again, at the laser powers which we used, the molecules are only excited to their first singlet or triplet states.

Although there is no general consensus concerning the mechanism responsible for laser damage of polymeric materials, there is already enough evidence to establish that the laser damage of polymers is energy

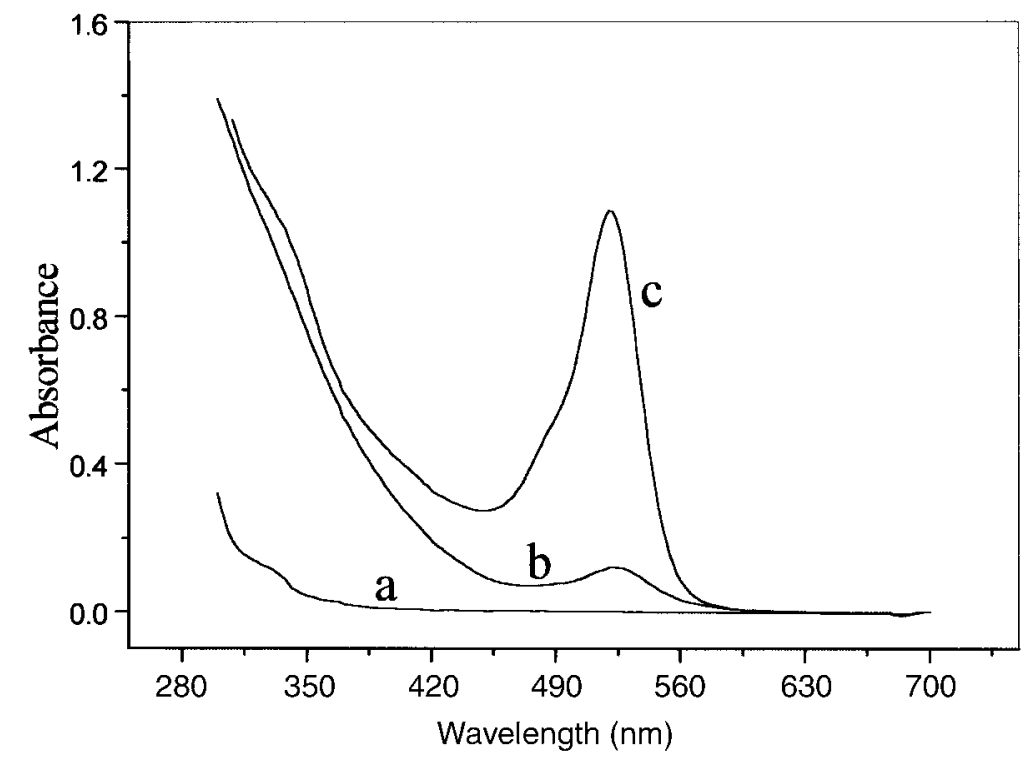

FIGURE 6 Absorption spectra of rhodamine 6G doped PMMA sample (a) plain PMMA (b) after bleaching (c) before bleaching. 
dependent and that the viscoelastic properties of the matrix determine damage resistance [33]. Pure polymers with saturated chains do not absorb in the ultraviolet-visible region of the spectrum (300-700 nm) (Fig. 6). Hence it is evident that the impurities act as photo sensitizers. It is believed that the dye molecules in any of these excited states react with the polymer molecules, or with the free radicals or with any other impurities in the sample (traces of transition metals, hydroperoxides, etc.), which are introduced during the synthesis or processing to give a colourless product. Dissolved oxygen and concentration of dye in the sample may also contribute to the reaction [34].

\section{CONCLUSION}

A dual beam thermal lens technique was successfully employed for the determination of absolute values of the fluorescence quantum yield of a dye doped polymer. The thermal lens method offers significant advantages over conventional methods for determining absolute values of quantum yields since no standard is necessary. The variation of quantum yield with concentration of Rhodamine $6 \mathrm{G}$ chromophore in polymer matrices is similar to that in liquid solution indicating that such systems are very attractive media for laser systems. In addition to this the high photostability of the dyes and the compactness of the samples, will greatly improve the possibilities for developing practical tunable solid-state dye lasers. The photostability depends on the chemical structure of macromolecules, the polymerization initiation procedure, concentration of dye in the sample, presence of oxygen in the initial monomer composition, sample temperature and thermal treatment.

A factor to be considered in the design of laser media is the dye concentration. The use of samples with high dye concentration can result in aggregation of the dye, which causes the fluorescence quenching of the monomer emission.

\section{Acknowledgements}

The authors are grateful to UGC (Govt. of India) for financial assistance. BP is thankful to the CSIR (Govt. of India) for financial help. The authors also acknowledge the financial support from the Netherlands University Federation for International Collaboration (NUFFIC). 


\section{References}

[1] Soffer, B. H. and McFarland, B. B. (1967) Appl. Phys. Lett., 10, 266.

[2] Peterson, O. G. and Snavely, B. B. (1968) Appl. Phys. Lett., 12, 238.

[3] Wang, H. and Gampel, L. (1976) Opt. Commun., 18, 444.

[4] Rodchenkova, V. V., Tsogoeva, S. A., Muraveva, T. M., Denisov, L. K. and Uzhinov, B. M. (1986) Opt. Spectrosc. (USSR), 60, 35.

[5] Reisfeld, R., Zusman, R., Cohen, Y. and Eyal, M. (1988) Chem. Phys. Lett., 147, 142.

[6] Hermes, R. E., Allik, T. H., Chandra, S. and Hutchinson, J. A. (1993) Appl. Phys. Lett., 63, 877.

[7] Costela, A., Florido, F., Garcia-Moreno, I., Duchowiz, R., Amat-Guerri, F., Figuera, J. M. and Sastre, R. (1995) Appl. Phys. B, 60, 383.

[8] Maslyukov, Sokolov, S., Kaivola, M., Nyholm, K. and Popov, S. (1995) Appl. Opt., 34, 1516.

[9] Kumar, N. D., Bhawalkar, J. D. and Prasad, P. N. (1997) Appl. Phys. Lett., 71, 999.

[10] Wadsworth, W. J., Giffin, S. M., Mckinnie, I. T., Sharpe, J. C., Woolhouse, A. D., Haskell, T. G. and Smith, G. J. (1999) Appl. Opt., 38, 2504.

[11] Yariv, E., Schultheiss, S., Saraidarov, T. and Reisfeld, R. (2001) Opt. Mater., 16, 29.

[12] Baesso, M. L., Bento, A. C., Andrade, A. A., Sampio, J. A., Pecoraro, E., Nunes, L. A. O., Catunda, T. and Gama, S. (1998) Phys. Rev. B, 57, 10545.

[13] Dyumaev, K. M., Manenkov, A. A., Maslyukov, A. P., Matyushin, G. A., Nechitailo, V. S. and Prokhorov, A. M. (1983) Sov. J. Quantum Electron, 13, 503.

[14] Demas, J. N. and Crosby, J. A. (1971) J. Phys. Chem., 75, 991.

[15] Gordon, J. P., Leite, R. C., Moore, R. S., Porto, P. S. and Whinnery, J. R. (1965) J. Appl. Phys., 36, 3.

[16] Terazima, M., Kanno, H. and Azumi, T. (1990) Chem. Phys. Let., 173, 327.

[17] Bindhu, C. V., Harilal, S. S., Kurian, A., Nampoori, V. P. N. and Vallabhan, C. P. G. (1998) J. Nonlinear Opt. Phys. \& Mats., 7, 531.

[18] Bindhu, C. V., Harilal, S. S., Nampoori, V. P. N. and Vallabhan, C. P. G. (1998) Opt. Eng., 37, 2791.

[19] Dovichi, N. J. and Harris, J. M. (1979) Anal. Chem., 51, 728.

[20] Franko, M. (2001) Talanta, 54, 1.

[21] Kurian, A., Unnikrishnan, K. P., Gopinath, P., Nampoori, V. P. N. and Vallabhan, C. P. G. (2001) J. Nonlinear Opt. Phys. \& Mats., 10, 415.

[22] Brannon, J. H. and Magde, D. (1978) J. Phys. Chem., 82, 705.

[23] Shen, J. and Snook, R. D. (1989) Chem. Phys. Lett., 155, 583.

[24] Lesiecki, M. L. and Drake, J. M. (1982) Appl. Opt., 21, 557.

[25] Grabiner, F. R., Siebert, D. R. and Flynn, G. W. (1972) Chem. Phys. Lett., 17, 189.

[26] Snook, R. D. and Lowe, R. D. (1995) Analyst, 120, 2051.

[27] Korobov, V. E., Shubin, V. V. and Chibisov, A. K. (1977) Chem. Phys. Lett., 45, 498.

[28] Korobov, V. E. and Chibisov, A. K. (1978) J. Photochem., 9, 411.

[29] Bindhu, C. V., Harilal, S. S. (2001) Analytical Science, 17, 141.

[30] El-Shahawy, M. A. (2000) Polymer Testing, 19, 821.

[31] Kurian, A., Unnikrishnan, K. P., Thomas Lee, S., Nampoori, V. P. N. and Vallabhan, C. P. G. Laser Chem. (in press).

[32] George, N. A., Aneeshkumar, B., Radhakrishnan, P. and Vallabhan, C. P. G. (1999) J. Phys. D: Appl. Phys., 32, 1745.

[33] Costela, A., Garcia-Moreno, I., Figuera, J. M., Amat-Guerri, F., Mallavia, R., SantaMaria, M. D. and Sastre, R. (1996) J. Appl. Phys., 80, 3167.

[34] Dyumaev, K. M., Manenkov, A. A., Maslyukov, A. P., Matyushin, G. A., Nechitailo, V. S. and Prokhorov, A. M. (1992) J. Opt. Soc. Am. B, 9, 143. 